\title{
Tandem Sequence of Cross Metathesis-Ring-Closing Metathesis Reaction of Alkynyl Silyloxy-Tethered Enynes
}

\author{
Sangho Park, Mansuk Kim and Daesung Lee* \\ Department of Chemistry, University of Wisconsin, Madison, WI 53706 \\ dlee@chem.wisc.edu
}

\section{Materials and Methods}

${ }^{1} \mathrm{H}$ NMR and ${ }^{13} \mathrm{C}$ NMR spectra were recorded on a Bruker AC-250 or a Bruker AC-300 spectrometer. Tetramethylsilane (TMS) or $\mathrm{CDCl}_{3}$ served as internal standard $C_{-}=0$ or 7.26 respectively) for ${ }^{1} \mathrm{H}$ NMR $\mathrm{CDCl}_{3}$ was used as internal standard ( $\left.=77.2\right)$ for ${ }^{13} \mathrm{C}$ NMR. All reactions were run under an atmosphere of nitrogen, unless otherwise indicated. Infrared spectra (IR) were obtained on a FT-IR equipped with a DTGS detector. Flasks were ovendried overnight and cooled under a stream of nitrogen. Compounds were purchased from Aldrich. Dichloromethane was distilled from $\mathrm{CaH}_{2}$. All other solvents were purified based on standard procedures. Flash chromatography was performed using silica gel $60 \AA$ (32-63 mesh) from Sorbent Technologies. Reactions were monitored by thin layer chromatography (TLC) using $0.25 \mathrm{~mm}$ E. Merk pre-coated silica gel 60 (particle size $0.040-0.063 \mathrm{~mm}$ ).

\section{Representative Procedure for a Tandem CM-RCM Reaction of 9a-f}

Silyated enyne 9b (34 mg, $0.101 \mathrm{mmol})$ and 5-hexenyl-1-acetate (57 mg, $0.401 \mathrm{mmol})$ were dissolved in freshly distilled $\mathrm{CH}_{2} \mathrm{Cl}_{2}(0.03 \mathrm{M})$. Grubbs $2^{\text {nd }}$ generation catalyst $\mathbf{1}$, $\left(\mathrm{H}_{2} \mathrm{Imes}\right)\left(\mathrm{PCy}_{3}\right) \mathrm{Cl}_{2} \mathrm{Ru}=\mathrm{CHPh},(6 \mathrm{mg}, 0.007 \mathrm{mmol})$ was added to the reaction mixture. After stirring under reflux for $4 \mathrm{~h}$, the reaction mixture was concentrated and the residue was purified by flash column chromatography (hexanes/EtOAc 20:1) to yield the desired CMRCM product $\mathbf{1 0 b}$ (oil, $21 \mathrm{mg}, 46 \%$ ). 


\section{Desilylation of 10a}

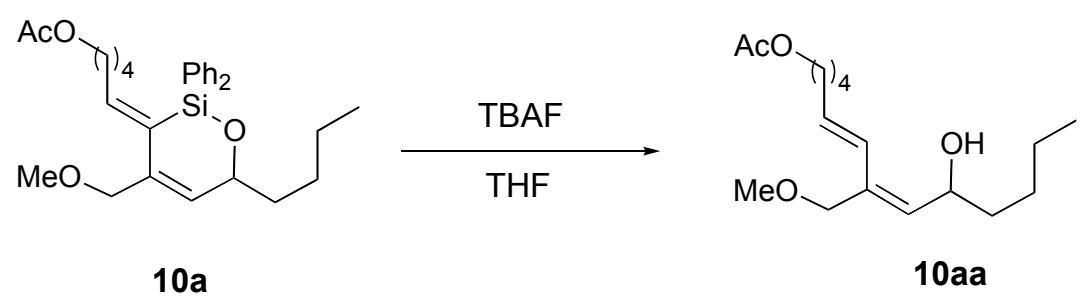

To a solution of compound $\mathbf{1 0 a}(35 \mathrm{mg}, 0.073 \mathrm{mmol})$ in THF, was added $n-\mathrm{BuN}_{4} \mathrm{~F}(1.0 \mathrm{M}$ in THF, $0.10 \mathrm{~mL}$ ) at room temperature. After stirring for $4 \mathrm{~h}$, the reaction mixture was concentrated under reduced pressure and the residue was directly purified by flash column chromatography (hexanes/EtOAc 5:1) to afford the desilylated product 10aa (oil, $21 \mathrm{mg}$, 96\%). ${ }^{1}$ H NMR (300 MHz, $\left.\mathbf{C D C l}_{3}\right):{ }_{-} 6.30(\mathrm{~d}, J=15.8 \mathrm{~Hz}, 1 \mathrm{H}), 5.84(\mathrm{dt}, J=15.8,7.0 \mathrm{~Hz}$, $1 \mathrm{H}), 5.47(\mathrm{~d}, J=8.8 \mathrm{~Hz}, 1 \mathrm{H}), 4.57(\mathrm{dt}, J=8.8,6.8 \mathrm{~Hz}, 1 \mathrm{H}), 4.06(\mathrm{t}, J=6.8 \mathrm{~Hz}, 2 \mathrm{H}), 4.03$ (m, 2H), $3.32(\mathrm{~s}, 3 \mathrm{H}), 2.16(\mathrm{q}, J=7.3 \mathrm{~Hz}, 3 \mathrm{H}), 2.04(\mathrm{~s}, 3 \mathrm{H}), 1.57-1.69$ (m, 4H, overlap), 1.42-1.54 (m, 4H, overlap), 1.22-1.40 (m, 4H, overlap), 0.89 (t, $J=6.8 \mathrm{~Hz}, 3 \mathrm{H}) ;{ }^{13} \mathbf{C}$ NMR (75 MHz, $\left.\mathbf{C D C l}_{3}\right)$ : _ 171.44, 134.52, 132.90, 132.76, 124.80, 74.63, 67.64, 64.53, 58.02, 37.43, 33.18, 28.29, 27.71, 25.68, 22.85, 21.20, 14.24; HRMS (ESI) calcd for $\mathrm{C}_{17} \mathrm{H}_{30} \mathrm{O}_{4} \mathrm{Na}$ $[\mathrm{M}+\mathrm{Na}]^{+}:$321.2042, found. 321.2037 .

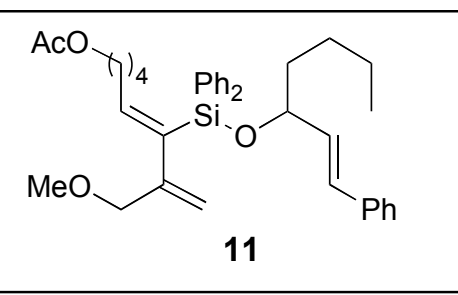

${ }^{1}$ H NMR (300 MHz, CDCl $)$ : _ $7.68(\mathrm{~m}, 5 \mathrm{H}), 7.37(\mathrm{~m}, 6 \mathrm{H})$, $7.24(\mathrm{~m}, 5 \mathrm{H}), 6.31(\mathrm{t}, J=7.5 \mathrm{~Hz}, 1 \mathrm{H}), 6.22(\mathrm{~d}, J=15.7 \mathrm{~Hz}$ $1 \mathrm{H}), 6.02(\mathrm{dd}, J=15.9,6.9 \mathrm{~Hz}, 1 \mathrm{H}), 4.96(\mathrm{~d}, J=2.0 \mathrm{~Hz}, 1 \mathrm{H})$, $4.92(\mathrm{~d}, J=2.0 \mathrm{~Hz}, 1 \mathrm{H}), 4.34(\mathrm{q}, J=6.4 \mathrm{~Hz}, 1 \mathrm{H}), 3.85(\mathrm{~s}$, $2 \mathrm{H}), 3.79(\mathrm{t}, J=6.6 \mathrm{~Hz}, 2 \mathrm{H}), 3.15(\mathrm{~s}, 3 \mathrm{H}), 2.02(\mathrm{t}, J=9.0 \mathrm{~Hz}$, 2H), $1.97(\mathrm{~s}, 3 \mathrm{H}), 1.66-1.41(\mathrm{~m}, 4 \mathrm{H}), 1.37-1.11(\mathrm{~m}, 6 \mathrm{H}), 0.89(\mathrm{~m}, 3 \mathrm{H}) ;{ }^{13} \mathbf{C}$ NMR (75 $\left.\mathbf{M H z}, \mathbf{C D C l}_{3}\right):$ _ $171.26,151.73,148.74,135.59,135.50,135.30,132.74,129.99,129.91$, $128.61,127.93,127.84,127.46,126.56,111.17,75.62,75.07,57.98,37.90,32.63$, 28.29, 27.27, 25.79, 25.69, 22.82, 21.17, 14.24; IR (thin film) 2927, 1739, 1429, 1240, $1114 \mathrm{~cm}^{-1}$; HRMS (EI) calcd for $\mathrm{C}_{37} \mathrm{H}_{46} \mathrm{O}_{4} \mathrm{Si}[\mathrm{M}]^{\circ+}: 582.3165$, found 582.3144. 


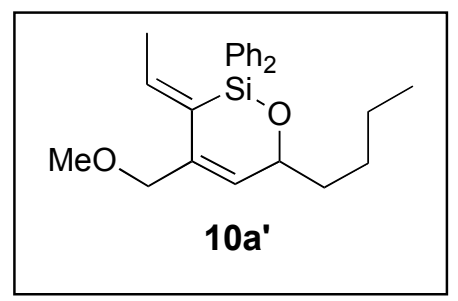

${ }^{1}$ H NMR (300 MHz, CDCl$\left.)_{3}\right):{ }_{-} 7.67(\mathrm{~m}, 4 \mathrm{H}), 7.41(\mathrm{~m}, 6 \mathrm{H})$, $6.64(\mathrm{q}, J=7.2 \mathrm{~Hz}, 1 \mathrm{H}), 5.73(\mathrm{~d}, J=2.8 \mathrm{~Hz}, 1 \mathrm{H}), 4.60$ (brq, $1 \mathrm{H}), 4.20(\mathrm{~m}, 2 \mathrm{H}), 3.36(\mathrm{~s}, 3 \mathrm{H}), 1.63(\mathrm{~d}, J=7.1 \mathrm{~Hz}, 3 \mathrm{H}), 1.14-$ $1.60(\mathrm{~m}, 6 \mathrm{H}), 0.83(\mathrm{t}, J=7.1 \mathrm{~Hz}, 3 \mathrm{H}) ;{ }^{13} \mathrm{C}$ NMR (75 MHz, $\left.\mathbf{C D C l}_{3}\right):{ }_{\text {- }} 137.41,135.42,135.36,132.01,130.22,128.39$, $128.12,128.03,75.33,72.75,57.85,38.31,27.54,22.78,19.87,14.21$; IR (thin film) 2923, 2174, 1430, 1373, $1114 \mathrm{~cm}^{-1}$; HRMS (EI) calcd for $\mathrm{C}_{24} \mathrm{H}_{30} \mathrm{O}_{2} \mathrm{Si}[\mathrm{M}]^{\circ+}: 378.2015$, found 378.1997.

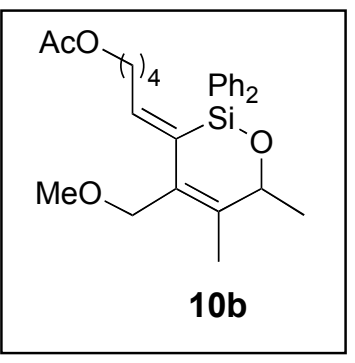

${ }^{1}$ H NMR (300 MHz, $\left.\mathbf{C D C l}_{3}\right):{ }_{-} 7.64(\mathrm{td}, J=7.7,1.5 \mathrm{~Hz}, 4 \mathrm{H}), 7.36$ $(\mathrm{m}, 6 \mathrm{H}), 6.52(\mathrm{t}, J=7.7 \mathrm{~Hz}, 1 \mathrm{H}), 4.56(\mathrm{q}, J=6.7 \mathrm{~Hz}, 1 \mathrm{H}), 4.27(\mathrm{~d}, J$ $=10.5 \mathrm{~Hz}, 1 \mathrm{H}), 4.16(\mathrm{~d}, J=10.5 \mathrm{~Hz}, 1 \mathrm{H}), 3.74(\mathrm{t}, J=6.3 \mathrm{~Hz}, 2 \mathrm{H})$, $3.36(\mathrm{~s}, 3 \mathrm{H}), 1.96(\mathrm{~s}, 3 \mathrm{H}), 1.93-2.01(\mathrm{~m}, 2 \mathrm{H}$, overlap), $1.81(\mathrm{~s}, 3 \mathrm{H})$, $1.31(\mathrm{~d}, J=6.7 \mathrm{~Hz}, 3 \mathrm{H}), 1.03-1.25(\mathrm{~m}, 6 \mathrm{H}) ;{ }^{13} \mathbf{C}$ NMR (75 MHz, $\left.\mathbf{C D C l}_{3}\right):$ _ $171.20,141.37,136.25,135.47,135.28,130.47,130.20$, $129.13,128.07,74.80,70.53,64.48,57.92,33.67,28.24,26.05,24.11,21.14,18.58$; IR (thin film) 2927, 1739, 1429, 1241, $1094 \mathrm{~cm}^{-1}$; HRMS (EI) calcd for $\mathrm{C}_{26} \mathrm{H}_{31} \mathrm{O}_{4} \mathrm{Si}$ [M$\left.\mathrm{CH}_{3},\right]^{++}: 435.1992$, found 435.1996 .

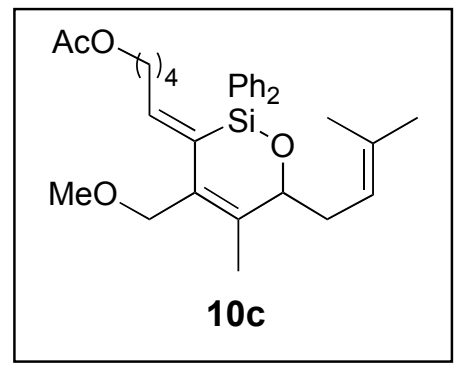

${ }^{1}$ H NMR (300 MHz, CDCl $)$ ) _ $7.63(\mathrm{~m}, 4 \mathrm{H}), 7.31-7.41(\mathrm{~m}$, $6 \mathrm{H}), 6.52(\mathrm{t}, J=7.7 \mathrm{~Hz}, 1 \mathrm{H}), 5.14(\mathrm{t}, J=6.7 \mathrm{~Hz}, 1 \mathrm{H}), 4.43(\mathrm{dd}$, $J=8.4,3.5 \mathrm{~Hz}, 1 \mathrm{H}), 4.29(\mathrm{~d}, J=10.6 \mathrm{~Hz}, 1 \mathrm{H}), 4.16(\mathrm{~d}, J=$ $10.6 \mathrm{~Hz}, 1 \mathrm{H}), 3.74(\mathrm{t}, J=6.5 \mathrm{~Hz}, 2 \mathrm{H}), 3.34(\mathrm{~s}, 3 \mathrm{H}), 2.36-2.47$ $(\mathrm{m}, 1 \mathrm{H}), 2.05-2.18(\mathrm{~m}, 1 \mathrm{H}), 1.96(\mathrm{~s}, 3 \mathrm{H}), 1.92-2.04(\mathrm{~m}, 2 \mathrm{H}$, overlap), $1.83(\mathrm{~s}, 3 \mathrm{H}), 1.40(\mathrm{~s}, 6 \mathrm{H}), 1.02-1.25(\mathrm{~m}, 2 \mathrm{H}), 1.31(\mathrm{~d}$, $J=6.7 \mathrm{~Hz}, 3 \mathrm{H}), 1.03-1.25(\mathrm{~m}, 5 \mathrm{H}), 0.89(\mathrm{~m}, 1 \mathrm{H}) ;{ }^{13} \mathbf{C} \mathbf{N M R}\left(\mathbf{7 5} \mathbf{M H z}, \mathbf{C D C l}_{3}\right)$ : 171.30 , $141.37,140.17,135.60,135.31,133.24,131.38,130.09,129.35,127.95,121.20,79.13$, $70.50,64.50,57.81,36.20,33.63,28.22,26.09,25.94,21.15,18.89,18.00$; HRMS (EI) calcd for $\mathrm{C}_{26} \mathrm{H}_{31} \mathrm{O}_{4} \mathrm{Si}\left[\mathrm{M}-\mathrm{C}_{4} \mathrm{H}_{7}\right]^{\bullet+}: 435.1992$, found 435.2011 . 


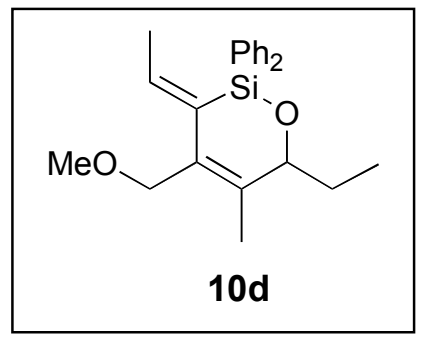

${ }^{1}$ H NMR (300 MHz, $\left.\mathbf{C D C l}_{3}\right)$ : _ 7.59-7.69 (m, 4H), 7.34-7.45 (m, 6H), 6.67 (q, $J=7.2 \mathrm{~Hz}, 1 \mathrm{H}), 4.29-4.33$ (m, 1H, overlap), 4.29 (d, $J=10.7 \mathrm{~Hz}, 1 \mathrm{H}$, overlap), $4.15(\mathrm{~d}, J=10.7 \mathrm{~Hz}, 1 \mathrm{H})$, $3.36(\mathrm{~s}, 3 \mathrm{H}), 1.82$ (s, 3H), 1.60-1.78 (m, 1H, overlap), 1.64 (d, $J$ $=7.2 \mathrm{~Hz}, 3 \mathrm{H}), 1.34-1.51(\mathrm{~m}, 1 \mathrm{H}), 0.81(\mathrm{t}, J=7.2 \mathrm{~Hz}, 3 \mathrm{H}) ;{ }^{13} \mathbf{C}$

NMR (75 MHz, CDCl $)$ : _ 139.96, 136.33, 136.16, 135.48, 135.31, 130.08, 129.92, 128.03, 80.26, 70.55, 57.90, 30.32, 20.01, 18.79, 10.49; HRMS (EI) calcd for $\mathrm{C}_{23} \mathrm{H}_{28} \mathrm{O}_{2} \mathrm{Si}[\mathrm{M}]^{++}: 364.1859$, found 364.1847 .
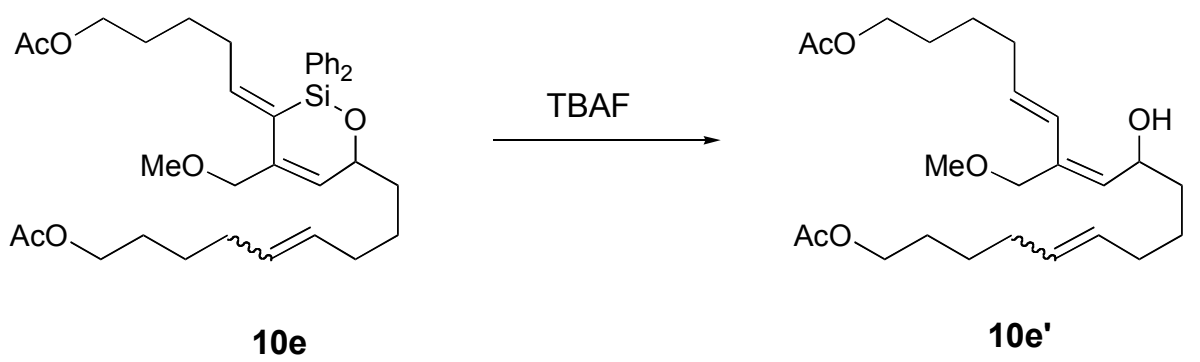

Compound 10e_. ${ }^{1} \mathbf{H}$ NMR $\left(250 \mathbf{~ M H z}, \mathbf{C D C l}_{3}\right)$ : _ $6.31(\mathrm{~d}, J=15.4 \mathrm{~Hz}, 1 \mathrm{H}), 5.85(\mathrm{dt}, J=$ 15.4, $7.3 \mathrm{~Hz}, 1 \mathrm{H}), 5.48(\mathrm{~d}, J=9.2 \mathrm{~Hz}, 1 \mathrm{H}), 5.38(\mathrm{~m}, 2 \mathrm{H}), 4.58(\mathrm{br} \mathrm{dt}, 1 \mathrm{H}), 4.00-4.10(\mathrm{~m}$, $6 \mathrm{H}$, overlap), $3.32(\mathrm{~s}, 3 \mathrm{H}), 1.95-2.21$ (m, 6H, overlap), 2.04(s, $6 \mathrm{H}), 1.21-1.70(\mathrm{~m}, 12 \mathrm{H}$, overlap) ; ${ }^{13} \mathbf{C}$ NMR (62 MHz, $\left.\mathbf{C D C l}_{3}\right)$ : _ 171.47, 134.70, 132.89, 132.73, 130.72, 129.77, $124.82,76.12,74.62,69.04,67.53,64.71,58.09,37.19,33.22,32.30,28.33,25.72,25.49$, 21.23; HRMS (ESI) calcd for $\mathrm{C}_{24} \mathrm{H}_{40} \mathrm{O}_{6} \mathrm{Na}[\mathrm{M}+\mathrm{Na}]^{+}: 447.2723$, found 447.2729.

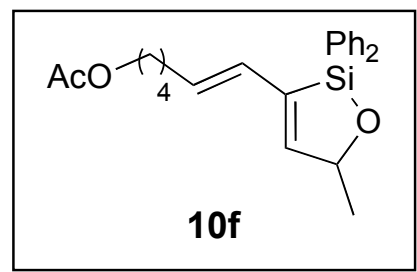

${ }^{1}$ H NMR (300 MHz, CDCl $)$ : _ 7.64-7.68 (m, 4H), 7.36-7.49 (m, 6H), $6.72($ brs, $1 \mathrm{H}), 6.40(\mathrm{~d}, J=16.0 \mathrm{~Hz}, 1 \mathrm{H}), 5.61(\mathrm{dt}, J=$ $15.5,6.9 \mathrm{~Hz}, 1 \mathrm{H}), 5.04$ (brq, $J=6.6 \mathrm{~Hz}, 1 \mathrm{H}), 3.99$ (t, $J=6.5 \mathrm{~Hz}$, 2H), 2.02-2.11 (m, 2H, overlap), 2.03 (s, 3H, overlap), 1.49-1.58 $(\mathrm{m}, 2 \mathrm{H}), 1.31-1.44(\mathrm{~m}, 2 \mathrm{H}) 1.42(\mathrm{~d}, J=6.7 \mathrm{~Hz}, 3 \mathrm{H}) ;{ }^{13} \mathbf{C} \mathbf{~ N M R}$

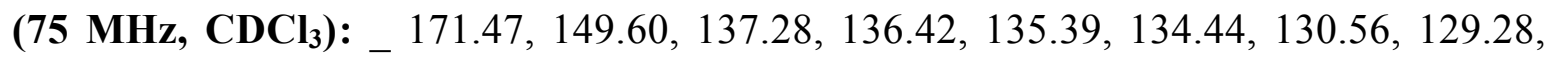
128.14, 78.59, 64.50, 32.59, 28.13, 25.61, 23.74, 21.19; HRMS (EI) calcd for $\mathrm{C}_{24} \mathrm{H}_{28} \mathrm{O}_{3} \mathrm{Si}$ $[\mathrm{M}]^{\circ+}: 392.1808$, found 392.1799 . 


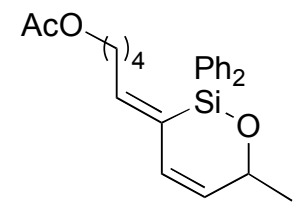

$10 \mathrm{~g}$

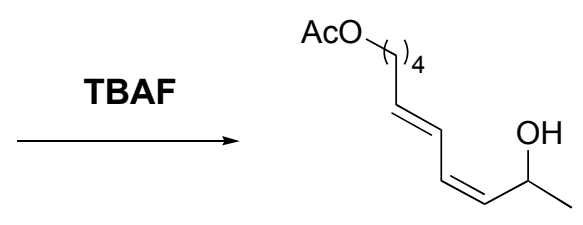

$10 g^{\prime}$

Compound 10g_. ${ }^{1} \mathbf{H}$ NMR (300 $\left.\mathbf{M H z}, \mathbf{C D C l}_{3}\right)$ : ${ }_{-} 6.35(\mathrm{dd}, J=15.2,12.3 \mathrm{~Hz}, 1 \mathrm{H}), 5.96$ (t, $J=10 \mathrm{~Hz}, 1 \mathrm{H}), 5.71(\mathrm{dt}, J=14.8,7.1 \mathrm{~Hz}, 1 \mathrm{H}), 5.34(\mathrm{dd}, J=9.8,9.7 \mathrm{~Hz}, 1 \mathrm{H}), 4.79(\mathrm{dq}, J$ $=8.7,6.3 \mathrm{~Hz}, 1 \mathrm{H}), 4.06(\mathrm{t}, J=6.6 \mathrm{~Hz}, 2 \mathrm{H}), 2.24(\mathrm{q}, J=7.2 \mathrm{~Hz}, 2 \mathrm{H}), 2.05(\mathrm{~s}, 3 \mathrm{H}), 1.58-1.70$ $(\mathrm{m}, 2 \mathrm{H}), 1.40-1.53(\mathrm{~m}, 2 \mathrm{H}), 1.28(\mathrm{~d}, J=6.3 \mathrm{~Hz}, 3 \mathrm{H}) ;{ }^{13} \mathbf{C} \mathbf{N M R}\left(62 \mathbf{M H z}_{\mathbf{C}} \mathbf{C D C l}_{3}\right)$ : $171.35,136.50,133.10,129.62,125.69,64.53$, 64.30, 32.53, 28.30, 25.66, 23.74, 21.23; IR (thin film) 3407, 2097, 1740, 1242, $1059 \mathrm{~cm}^{-1}$; HRMS (ESI) calcd for $\mathrm{C}_{12} \mathrm{H}_{20} \mathrm{O}_{3} \mathrm{Na}$ $[\mathrm{M}+\mathrm{Na}]^{++}: 235.1310$, found 235.1308 .

\section{Reaction of 2a with cis-2-Butene}

Silyated enyne $\mathbf{2 a}(37 \mathrm{mg}, 0.102 \mathrm{mmol})$ was dissolved in freshly distilled $\mathrm{CH}_{2} \mathrm{Cl}_{2}(0.03 \mathrm{M})$. Grubbs $2^{\text {nd }}$ generation catalyst $1(9 \mathrm{mg}, 0.011 \mathrm{mmol})$ was added to the reaction mixture. cis-2-Butene was added by bubbling into the solution throughout the reaction. After stirring under reflux for $6 \mathrm{~h}$, the reaction mixture was concentrated and the residue was purified by flash column chromatography (hexanes/EtOAc 20:1) to yield the desired CM-RCM product 12 (oil, $18 \mathrm{mg}, 42 \%$ ).

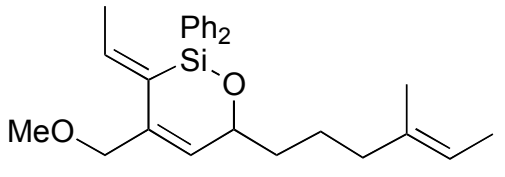

12
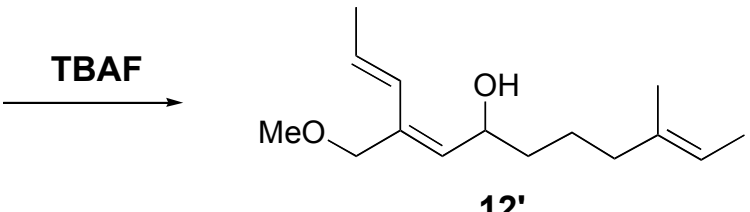

12

Compound 12_. ${ }^{1} \mathbf{H}$ NMR (300 MHz, $\left.\mathbf{C D C l}_{3}\right):$ _ $6.30(\mathrm{~d}, J=17.1 \mathrm{~Hz}, 1 \mathrm{H}), 5.90$ (dq, $J=$ 15.9, $6.6 \mathrm{~Hz}, 1 \mathrm{H}), 5.45$ (d, $J=8.9 \mathrm{~Hz}, 1 \mathrm{H}), 5.20$ (brs, 1H), 4.59 (brs, 1H), 4.06 (d, $J=11.7$ $\mathrm{Hz}, 1 \mathrm{H}), 4.00$ (d, $J=11.7 \mathrm{~Hz}, 1 \mathrm{H}), 3.32$ (s, 3H), 2.02 (dt, $J=16.0,6.4 \mathrm{~Hz}, 2 \mathrm{H}), 1.81$ (dd, $J$ $=6.6,1.8 \mathrm{~Hz}, 3 \mathrm{H}), 1.39-1.70(\mathrm{~m}, 7 \mathrm{H}), 1.57$ (s, 3H, overlap); ${ }^{13} \mathbf{C}$ NMR (75 MHz, CDCl $\left.\mathbf{3}\right)$ : _ 134.73, 132.38, 128.44, 125.61, 119.46, 118.84, 74.74, 67.59, 58.10, 39.66, 37.21, 23.76, 19.18, 15.65, 13.54; HRMS (EI) calcd for $\mathrm{C}_{15} \mathrm{H}_{26} \mathrm{O}_{2}[\mathrm{M}]^{{ }^{+}}:$: 238.1933, found 238.1939.

CM-RCM Reaction of 2a with 1,5-Cyclooctadiene 
Silyated enyne $\mathbf{2 a}(52 \mathrm{mg}, 0.143 \mathrm{mmol})$ and 1,5-cyclooctadiene (31 $\mathrm{mg}, 0.287 \mathrm{mmol})$ were dissolved in freshly distilled $\mathrm{CH}_{2} \mathrm{Cl}_{2}(0.03 \mathrm{M})$. Grubbs $2^{\text {nd }}$ generation catalyst $1(10 \mathrm{mg}$, $0.012 \mathrm{mmol}$ ) was added to the reaction mixture. After stirring under reflux for $6 \mathrm{~h}$, the reaction mixture was concentrated and the residue was purified by flash column chromatography (hexanes/EtOAc 20:1) to yield the desired CM-RCM product 13 (oil, 32 $\mathrm{mg}, 54 \%$ ).

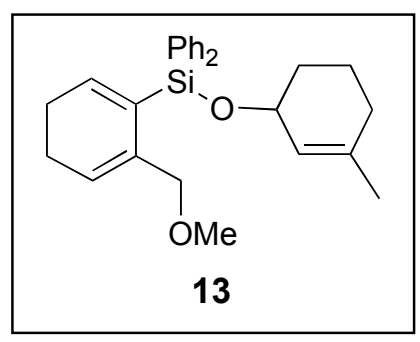

${ }^{1}$ H NMR (300 MHz, CDCl $)$ : _ $7.64-7.71(\mathrm{~m}, 4 \mathrm{H}), 7.33-7.44$ $(\mathrm{m}, 6 \mathrm{H}), 6.46(\mathrm{~m}, 1 \mathrm{H}), 5.90(\mathrm{~m}, 1 \mathrm{H}), 5.39(\mathrm{~m}, 1 \mathrm{H}), 4.41(\mathrm{~m}, 1 \mathrm{H})$, $3.85(\mathrm{~s}, 2 \mathrm{H}), 2.95(\mathrm{~s}, 3 \mathrm{H}), 2.15(\mathrm{~m}, 4 \mathrm{H}), 1.40-2.10(\mathrm{~m}, 6 \mathrm{H}$, overlap), 1.64 (s, 3H, overlap); ${ }^{13}$ C NMR (75 MHz, CDCl $)$ : $144.49,137.45,136.28,136.13,135.61,132.66,129.65,127.75$, $125.24,122.67,74.82,68.01,57.74,32.04,30.26,23.86,23.83$, 21.85, 19.74; IR (thin film) 3050, 2932, 1429, 1265, 1114, $909 \mathrm{~cm}^{-1}$; HRMS (ESI) calcd for $\mathrm{C}_{27} \mathrm{H}_{32} \mathrm{O}_{2} \mathrm{SiNa}[\mathrm{M}+\mathrm{Na}]^{+}: 439.2069$, found 439.2064.

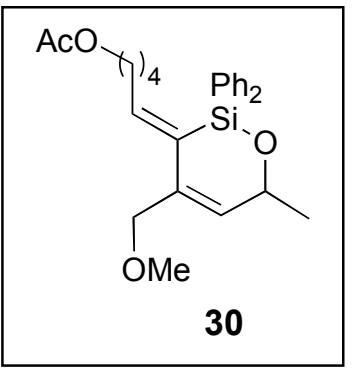

${ }^{1}$ H NMR (300 MHz, $\left.\mathbf{C D C l}_{3}\right):$ _ $7.63-7.67(\mathrm{~m}, 4 \mathrm{H})$, 7.34-7.48 (m, $6 \mathrm{H}), 6.48(\mathrm{t}, J=7.8 \mathrm{~Hz}, 1 \mathrm{H}), 5.73(\mathrm{~d}, J=2.2 \mathrm{~Hz}, 1 \mathrm{H}), 4.75(\mathrm{q}, J=$ $6.4 \mathrm{~Hz}, 1 \mathrm{H}), 4.19$ (s, 2H), $3.74(\mathrm{t}, J=6.4 \mathrm{~Hz}, 2 \mathrm{H}), 3.36(\mathrm{~s}, 3 \mathrm{H}), 1.94-$ 2.01(m, 2H, overlap), 1.97 (s, 3H), $1.32(\mathrm{~d}, J=6.7 \mathrm{~Hz}, 3 \mathrm{H}), 1.00-$ 1.21(m, 4H); ${ }^{13}$ C NMR (75 MHz, CDCl $)$ : _ 171.34, 142.19, $135.38,134.62,133.08,130.35,128.17,128.07,127.39,75.04$,

69.07 ,

64.42, 57.92, 33.70, 28.19, 25.81, 24.90, 21.17; HRMS (EI) calcd for $\mathrm{C}_{26} \mathrm{H}_{32} \mathrm{O}_{4} \mathrm{Si}[\mathrm{M}]^{\bullet+}$ : 436.2070, found 436.2087 .

\section{Representative Procedure for a CM Reaction of 49a-f}

Silyated enyne 49d (33 mg, $0.102 \mathrm{mmol})$ and 5-hexenyl-1-acetate (57 mg, $0.401 \mathrm{mmol})$ were dissolved in freshly distilled $\mathrm{CH}_{2} \mathrm{Cl}_{2}(0.03 \mathrm{M})$. Grubbs $2^{\text {nd }}$ generation catalyst 1 (10 $\mathrm{mg}, 0.012 \mathrm{mmol}$ ) was added to the reaction mixture. After stirring under reflux for $4 \mathrm{~h}$, the reaction mixture was concentrated and the residue was purified by flash column chromatography (hexanes/EtOAc 20:1) to yield the desired CM product 50d (oil, $38 \mathrm{mg}$, $82 \%)$. 


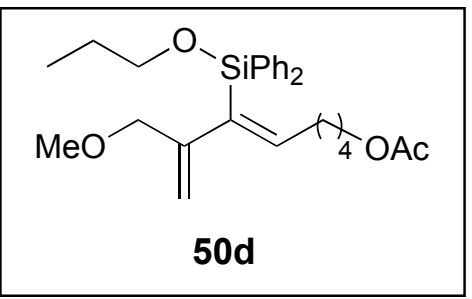

${ }^{1}$ H NMR (300 MHz, CDCl $)$ : _ 7.65-7.68 (m, 4H), 7.35-7.45 (m, 6H), $6.34(\mathrm{t}, J=7.7 \mathrm{~Hz}, 1 \mathrm{H}), 4.95$ (brs, $1 \mathrm{H}), 4.93$ (brs, $1 \mathrm{H}), 3.84(\mathrm{t}, J=6.4 \mathrm{~Hz}, 2 \mathrm{H}), 3.83(\mathrm{~s}, 2 \mathrm{H}), 3.62(\mathrm{t}, J=6.6 \mathrm{~Hz}$, 2H), 3.17 (s, 3H), 2.04 (m, 2H, overlap), 1.99 (s, 3H), 1.53 (sextet, $J=7.1 \mathrm{~Hz}, 2 \mathrm{H}), 1.15-1.38(\mathrm{~m}, 4 \mathrm{H}), 0.85(\mathrm{t}, \mathrm{J}=7.3$ $\mathrm{Hz}, 3 \mathrm{H}) ;{ }^{13} \mathbf{C}$ NMR (75 MHz, $\left.\mathbf{C D C l}_{3}\right):$ 171.29, 151.40, 148.92, 137.64, 135.32, 129.97, 127.96, 111,19, 75.52, 65.56, 64.51, 57.94, 32.45, 28.33, 25.84, 25.80, 21.13, 10.56; HRMS (ESI) calcd for $\mathrm{C}_{27} \mathrm{H}_{36} \mathrm{O}_{4} \mathrm{SiNa}[\mathrm{M}+\mathrm{Na}]^{+}: 475.2281$, found 475.2258 .

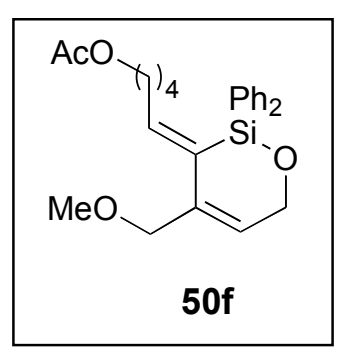

${ }^{1}$ H NMR (300 MHz, $\left.\mathbf{C D C l}_{3}\right)$ : _ 7.63-7.67 (m, 4H), 7.36-7.48 (m, $6 \mathrm{H}), 6.49(\mathrm{t}, J=7.7 \mathrm{~Hz}, 1 \mathrm{H}), 5.79(\mathrm{t}, J=3.2 \mathrm{~Hz}, 1 \mathrm{H}), 4.62(\mathrm{~s}, 2 \mathrm{H})$, $4.20(\mathrm{~d}, J=1 \mathrm{~Hz}, 2 \mathrm{H}), 3.74(\mathrm{t}, J=6.4 \mathrm{~Hz}, 2 \mathrm{H}), 3.36(\mathrm{~s}, 3 \mathrm{H}), 1.95-$ 2.07(m, 2H, overlap), 1.97 (s, 3H), 1.04-1.25(m, 4H); ${ }^{13} \mathbf{C}$ NMR (62 $\left.\mathbf{M H z}, \mathbf{C D C l}_{3}\right): 171.35,142.22,135.47,135.34,134.62,134.54$, $130.64,130.49,128.17,74.93,64.39,63.60,57.88,33.67,28.18$, 25.82, 21.13; HRMS (ESI) calcd for $\mathrm{C}_{25} \mathrm{H}_{30} \mathrm{O}_{4} \mathrm{SiNa}[\mathrm{M}+\mathrm{Na}]^{+}: 445.1811$, found 445.1832. 